\title{
Interscalene Versus Supraclavicular Approach to Brachial Plexus Nerve Blockade and Local Anesthetic Volume Effect on the Incidence of Phrenic Nerve Dysfunction during Shoulder Surgery
}

\author{
Hesham M Ezz MBChB', Anna F Dikstein MS-32, Hassan Rayaz $M D^{1}$, Elissa Mastrangelo MS, $B C^{1}$, \\ Michael J Medvecky MD', Thomas M. Halaszynski DMD, MD, MBA ${ }^{1 *}$ and Aymen A Alian MD ${ }^{1}$ \\ ${ }^{1}$ Department of Anesthesiology, Yale University, School of Medicine, USA \\ ${ }^{2}$ SUNY Downstate Medical College, New York \\ ${ }^{3}$ Department of Orthopedic surgery, Yale University, School of medicine, USA

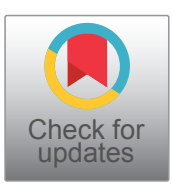

\begin{abstract}
Introduction: Interscalene (ISB) and Supraclavicular (SCB) brachial plexus blockade have been shown to be safe and effective for perioperative analgesia during shoulder surgery. However, potential adverse effects, including phrenic nerve dysfunction, are usually well tolerated by most patients but may be more compromising in those with limited respiratory reserve. This investigation examined effects of local anesthetic (LA) volumes and incidence of phrenic nerve influences (primary outcome) during ISB and SCB.

Methods: Observational study of 155 patients scheduled for shoulder surgery received incentive spirometry (IS; measuring inspiratory flow rates) to evaluate phrenic nerve influences from either ISB or SCB while using different LA volumes (> or $\leq 20 \mathrm{ml}$ ) prior to and following (20 minutes later) brachial plexus nerve blockade.

Results: Phrenic nerve dysfunction and compromise was $68.7 \%$ and $26.1 \%$ due to performance of an ISB and SCB respectively $(p<$ $0.001)$. When the ISB group was further divided according to the local anesthetic volume administered, the incidence of phrenic nerve dysfunction was $79 \%$ and $55.2 \%$ in high $(>20 \mathrm{ml})$ and low $(\leq 20 \mathrm{ml})$ local volume subgroups respectively, $(p=0.038)$. In the SCB group, the incidence of phrenic nerve dysfunction was $35.9 \%$ and $16.3 \%$ in high and low local anesthetic volume subgroups respectively, $(p=$ $0.035)$. There were no significant differences between LA volumes and block duration/efficacy in either the ISB $\left(R^{2}=0.0004\right)$ or SCB $\left(R^{2}=\right.$ 0.0081 ) approaches and identical/very similar quality of perioperative pain medicine was the same in both groups and in all 4 subgroups. Discussion: Statistically significant increases in phrenic nerve dysfunction occurred during ISB when compared to SCB and negative phrenic nerve influences were greater with LA volumes > $20 \mathrm{ml}$. ALL groups showed NO differences between LA volume and duration/efficacy of nerve blockade.

\section{Key points}

Question: [How to minimize the incidence of phrenic nerve dysfunction during preoperative brachial plexus (interscalene versus supraclavicular) blockade?.]

Findings: [Interscalene blockade results in a significantly higher incidence of phrenic nerve dysfunction when compared to effects from a supraclavicular approach nerve block. In addition, using local anesthetic volumes more than $20 \mathrm{ml}$ resulted in a higher incidence of phrenic nerve dysfunction, regardless of approach to the brachial plexus when compared to using volumes of $20 \mathrm{ml}$ or less. There were no differences in brachial plexus blockade efficacy or duration of effect between the 2 approaches and using greater or less than20ml volumes of local anesthetic.]

Meaning: [Preoperative peripheral nerve block for pain management during shoulder surgery can be achieved successfully using a supraclavicular approach to the brachial plexus using local anesthetic volumes of $20 \mathrm{ml}$ or less resulting in a lower incidence of side effects and without compromising either the quality or the duration of the nerve block.]
\end{abstract}

\section{Introduction}

Regional anesthesia (RA) continues to play an expanding role in all upper extremity surgical procedures. Specific approaches and techniques of brachial plexus nerve blockade results ineffective perioperative anesthesia/ analgesia. Performing a preoperative brachial plexus nerve block can result in lower intraoperative general anesthesia (GA) requirements, allows for fewer intraoperative narcotic boluses, and reduces or eliminates postoperative opioid analgesic rescue requirements [1]. Various approaches to
*Corresponding author: Thomas Halaszynski, Department of Anesthesiology, Yale University, School of Medicine, 20 Linden Shores, Branford, CT, 06405, USA, Tel: 203 785-2804

Accepted: December 23, 2021

Published online: December 27, 2021

Citation: Hesham ME, Dikstein AF, Rayaz H, et al. (2021) Interscalene Versus Supraclavicular Approach to Brachial Plexus Nerve Blockade and Local Anesthetic Volume Effect on the Incidence of Phrenic Nerve Dysfunction during Shoulder Surgery. J Clin Anesth Pain Manag 5(2):252-260 
Citation: Hesham ME, Dikstein AF, Rayaz H, et al. (2021) Interscalene Versus Supraclavicular Approach to Brachial Plexus Nerve Blockade and Local Anesthetic Volume Effect on the Incidence of Phrenic Nerve Dysfunction during Shoulder Surgery. J Clin Anesth Pain Manag 5(2):250-260

the brachial plexus have been reported that can deliver local anesthetics (LA) to target nerves including: interscalene (ISB), supraclavicular (SCB), infraclavicular (ICB) and axillary $(A B)$ nerve block approaches [2]. The most commonly performed brachial plexus approach during shoulder surgery has been the ISB [3].

Complications from brachial plexus nerve blockade can include vascular injury, pneumothorax, allergic reactions, and LA systemic toxicity to identify several. Another common adverse effect while performing proximal (i.e. ISB and SCB) brachial plexus nerve blockade is phrenic nerve influences from the injected LA that can result in a hemi-diaphragm (usually temporary).There have also been reports of chronic or long-term phrenic nerve dysfunction, including permanent phrenic nerve injury [4-6].These reports are likely secondary to multiple factors that can include direct neurotoxicity from the LA administered, perineural inflammation, scar tissue formation, adhesions that can form around the nerve, along with patient positioning in a beach chair position (independent of administering regional anesthesia for total shoulder arthroplasty) [4-6].

Ventral branches of cervical spinal nerves C5 to T1 form the brachial plexus and the phrenic nerve originates from C3-5 spinal nerves. Phrenic nerve usually descends on the scalenus anterior muscle separated from the brachial plexus by a fascia sheath placing it at variable degrees of risk to influences of LA injected during nerve/plexus blockade [7]. Historically, incidence of phrenic nerve influences are as high as $67 \%$ from a SCB and up to $100 \%$ with an ISB [8,9], but maybe reduced with ultrasound-guided (US) brachial plexus nerve blockade $[10,11]$. Respiratory complications from hemidiaphragm dysfunction can include pulmonary function testing compromise such as $26 \%$ reductions of forced expiratory volume in 1 second and $27 \%$ reductions in forced vital capacity [12]. These negative respiratory influences are usually well tolerated by healthy patients [13], but can have detrimental effects in those with limited respiratory reserve such as patients with chronic obstructive pulmonary disease, chronic smoking, those with obstructive sleep apnea and/or obesity hypoventilation syndrome [14].

Attempts to reduce the incidence of phrenic nerve dysfunction include: Alterations in brachial plexus block (location within the neurovascular bundle; i.e. anterior/ medial versus posterior/lateral needle tip placement) needle placement techniques $[15,16]$, administering lower concentrations/volume of LA $[17,18]$, altering the approach used (ISB versus SCB) when performing a brachial plexus block $[18,19]$, applying pressure cephalad to the injection site during LA administration [20], and relying on ultrasound (US)-guided RA to visualize (real-time) the injected LA spread $[10,12]$. These maneuvers have had degrees of success, but not reliably nor on a consistent basis. However, this study has objectively examined the influences and effect of different volumes of LA on phrenic nerve function during either an ISB or SCB approach to the brachial plexus block. Secondary outcomes of the investigation included measuring analgesic block duration and blockade efficacy. The purpose was to introduce a consistent and reliable approach to brachial plexus blockade for shoulder surgery that could provide postoperative analgesia with a statistically lowered incidence of deleterious phrenic nerve influences and dysfunction.

\section{Methods}

Following Institutional Review Board (IRB) approval[Human Investigation Committee (HIC) protocol number $\# 1205010184]$ of this non-randomized observational study, both written and verbal consents were obtained from 155patients scheduled for shoulder surgery (i.e. arthroscopy, rotator cuff repair, total shoulder replacement) at Yale New Haven Hospital (YNHH), New Haven, CT. Patients received a preoperative brachial plexus block with either a supraclavicular or interscalene approach to the brachial plexus for the primary purposes of providing postoperative analgesia and to minimize perioperative use of opiate analgesics. As all patients also received intraoperative general anesthesia (GA), performing a preoperative brachial plexus blockade also served as an additional adjuvant to minimize amounts/requirements of intraoperative GA. Exclusion criteria included upper extremity surgery other than shoulder surgery, altered mental status, age $<18$ years of age, history of neuropathic or myopathic disease, history of allergy to amide LA medications, systemic infections, or infection at the site of planned needle insertion. Patients were admitted to the Regional Anesthesia/Acute Pain Medicine (RAAPM) holding area and had their preoperative assessments (anesthesia, nursing and surgical) performed. Patients were interviewed, regional anesthesia and intraoperative anesthesia instructions discussed, study patients were then consented to receive a preoperative brachial plexus nerve block. The approach to the brachial plexus nerve block (interscalene versus a supraclavicular approach) in addition to the volume of LA injected was determined by the practitioner's experience and subjective preference ( $s$ ) in combination with patient body habitus, and also based on the patient's comorbidities along with observational US examination of the patient's brachial plexus anatomy.

Preoperative patient variables compiled and recorded included demographic data, history of present surgical and medical illnesses (medical comorbidities), current medications, along with the existence and/or severity of pain using an 11-point visual analogue scale for pain (VAS pain; 0-10 scale)both at rest and during movement. A pre-block or baseline incentive spirometry (IS) trial (i.e. 12 attempts performed pre- and then 12 attempts post-block) was performed after providing patient education, instruction, and practice attempts on the proper use of the IS device (Figure 1, described IS device). The number of balls elevated (i.e. 1-thru3 ) by the patient during each of the 12 attempts of the IS trial were counted and tabulated. The negative airway pressure generated by the patient with inspiration efforts during these trials was also monitored using a pressure transducer device (ICU Medical, San Clemente, CA 92673, USA) that is depicted in Figure 1.

All patients were monitored using pulse oximetry (Nellcor $^{\mathrm{TM}} \mathrm{SpO}_{2}$ adhesive sensor, Medtronic, MN, USA), electrocardiogram (EKG), and noninvasive blood pressure. 
Citation: Hesham ME, Dikstein AF, Rayaz H, et al. (2021) Interscalene Versus Supraclavicular Approach to Brachial Plexus Nerve Blockade and Local Anesthetic Volume Effect on the Incidence of Phrenic Nerve Dysfunction during Shoulder Surgery. J Clin Anesth Pain Manag 5(2):250-260

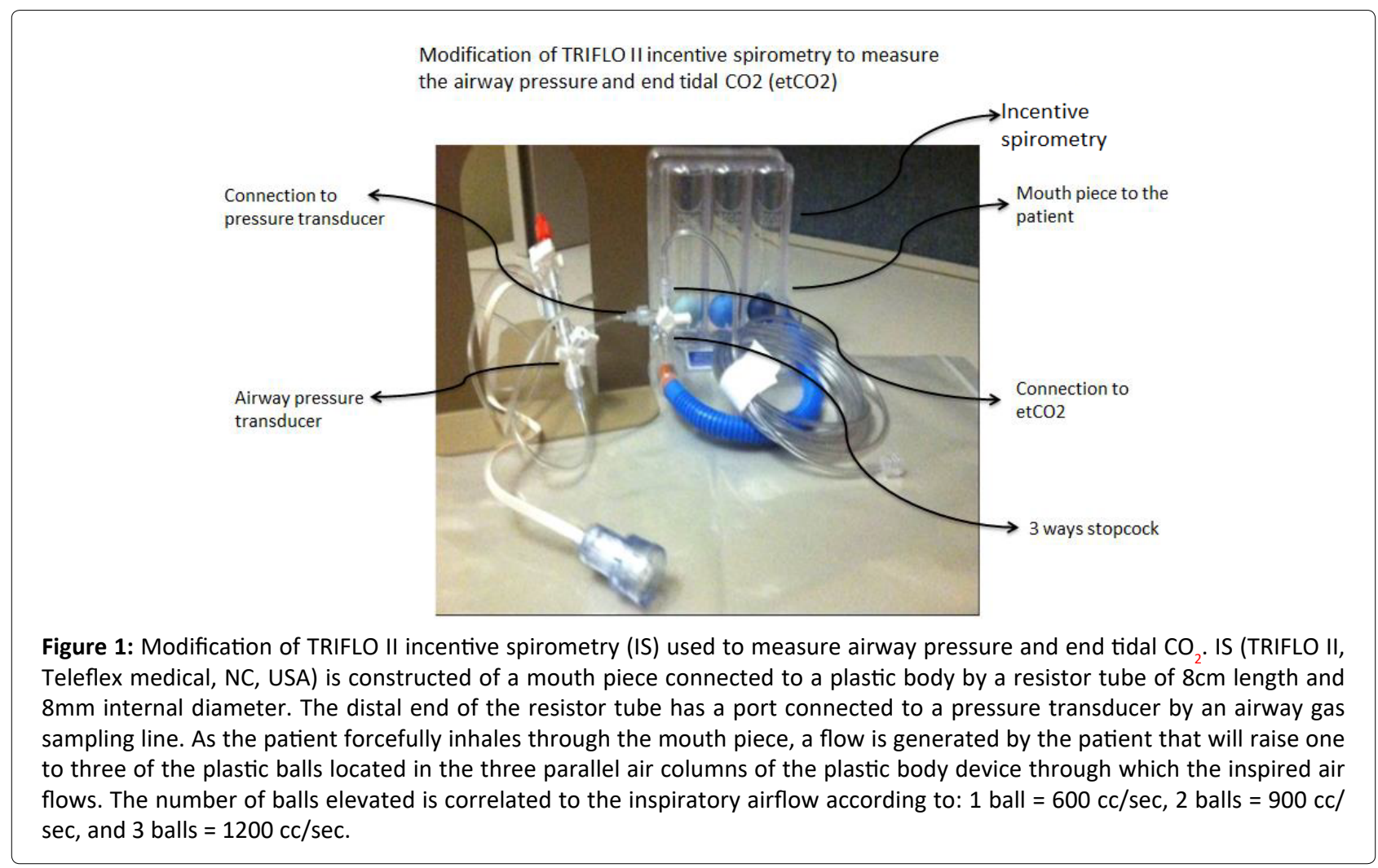

Intravenous (IV) access was obtained and oxygen administered by nasal cannula at a flow rate of $2 \mathrm{~L} / \mathrm{min}$. An institutional/hospital protocol "time out" was performed that included confirming patient's name, date of birth, medical record number, confirmation and marking of the site and side of surgery. Any necessary pre-block sedation was administered using midazolam (1-2mg) and/or fentanyl (50$100 \mathrm{mcg}$ ) as needed. The anticipated block site was cleaned with a chlorhexidine skin preparation solution (CloraPrep, Carefusion, NJ, USA) prior to an ultrasound-guided (US; Fujifilm, Sonosite, WA, USA) examination of the intended (ISB or SCB) brachial plexus block. Each study patient had the US probe initially positioned in the supraclavicular fossa to visualize the first rib, subclavian artery and brachial plexus (i.e. typically the divisions) lateral and posterior to the artery (Figure 2-right). Practitioners then scanned cephalad, following the hypoechoic nerve structures as they would most often be positioned (i.e. typically as 3 trunks) and visualized between the scalene muscles (Figure 2-left). At the level of the selected plexus block injection site (ISB or SCB approach), $1-2 \mathrm{ml}$ of $1 \%$ lidocaine skin infiltration was provided and then a $22 \mathrm{G}, 5 \mathrm{~cm}$ block-needle (Stimuplex $A^{\circ}$, B.Braun, USA) connected to a nerve stimulator (Stimuplex HNS12 with SENSe, B. Braun, USA) was introduced under direct in-plane US visualization until the block needle tip was placed next to the intended target site. The nerve stimulator current was then activated and milliamperes ( $\mathrm{mA}$ ) was slowly increased as well as any necessary block needle adjustments to assure lack of a motor response below $0.2 \mathrm{~mA}$. Following a trial of negative aspiration to reduce the potential of an IV injection, pre-determined volume $(\mathrm{s})$ of $0.5 \%$ ropivacaine was injected in divided aliquots (i.e. 2-3 ml; each aliquot was followed by repeat aspirations to reduce consequences of IV injection) under direct US visualization to assure spread of the LA around the intended targeted site of the nerve plexus. Subsequent to local anesthetic administration, the block needle was withdrawn, and the US probe then scanned/ positioned between the respective supraclavicular and interscalene anatomical areas of the brachial plexus in order to observe evidence of appropriate LA spread. An US image/ picture of the LA spread around each intended nerve plexus target site was taken and digitally recorded in the patients' medical records. All patients continued to be monitored for an additional 20 minutes following each block prior to entering the operating arena.

Subsequent to the 20-minute monitoring period, efficacy of the nerve block was tested by comparing shoulder sensory responses in the blocked extremity to alcohol swabs and pinprick compared to the contralateral limb. Accordingly, the complete absence of sensation (temperature and pinprick discrimination/evidence/change) within the blocked extremity was considered a successful block. A repeat trial of 12 IS attempts/breaths was conducted to measure the number of IS balls (i.e. 1, 2, 3 or portion thereof) elevated during forceful deep breathing and to measure the negative airway pressure generated subsequent to brachial plexus nerve blockade. The post nerve block IS data values and measurements were compared to the pre-nerve block IS trials. Each patient served as their own control and as an example, data collected from one study patient is depicted in Figure $3 \mathrm{~A}$.

The primary outcome data of this investigation was to determine and analyze the incidence of phrenic nerve dysfunction subsequent to performing a brachial plexus block in patients scheduled for shoulder surgery. Phrenic 
Citation: Hesham ME, Dikstein AF, Rayaz H, et al. (2021) Interscalene Versus Supraclavicular Approach to Brachial Plexus Nerve Blockade and Local Anesthetic Volume Effect on the Incidence of Phrenic Nerve Dysfunction during Shoulder Surgery. J Clin Anesth Pain Manag 5(2):250-260

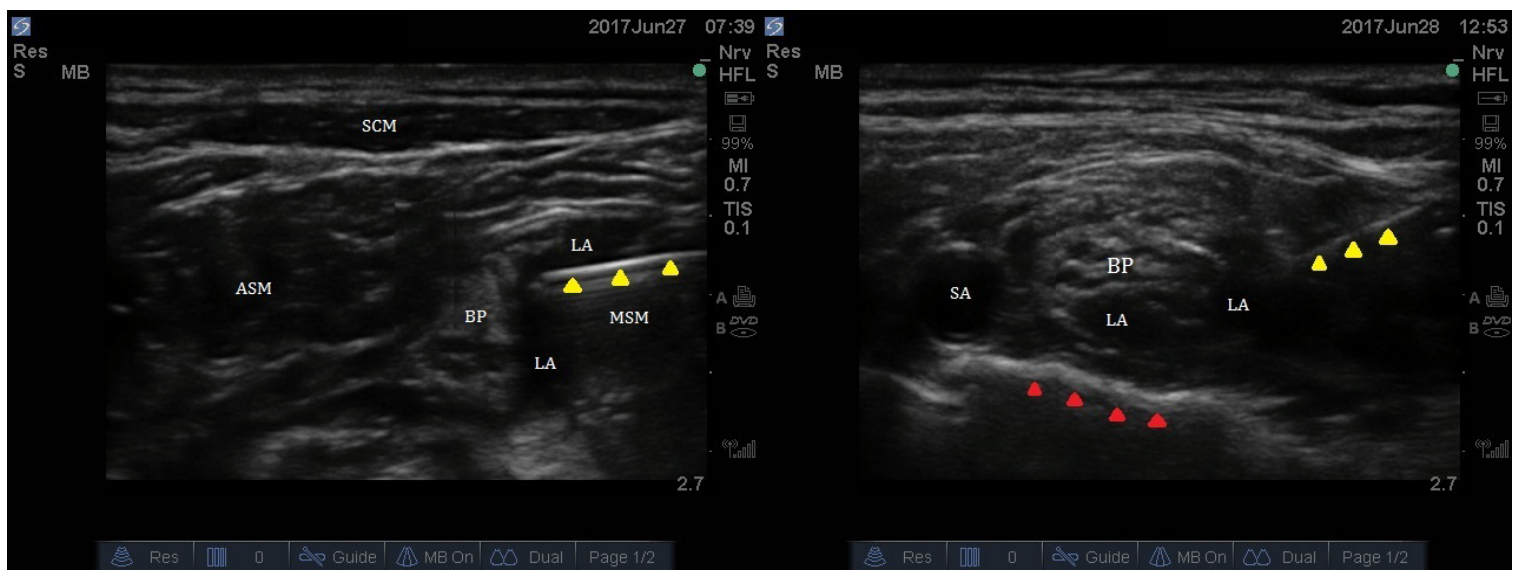

Figure 2: Ultrasound image of the brachial plexus anatomy, needle placement and LA spread at the level of interscalene block (left) and supraclavicular block (right). Yellow arrows: Block needle; Red arrows: First rib; BP: Brachial Plexus; LA: Local Anesthetic; SA: Subclavian Artery; ASM: Anterior Scalene Muscle; and MSM: Middle Scalene Muscle.
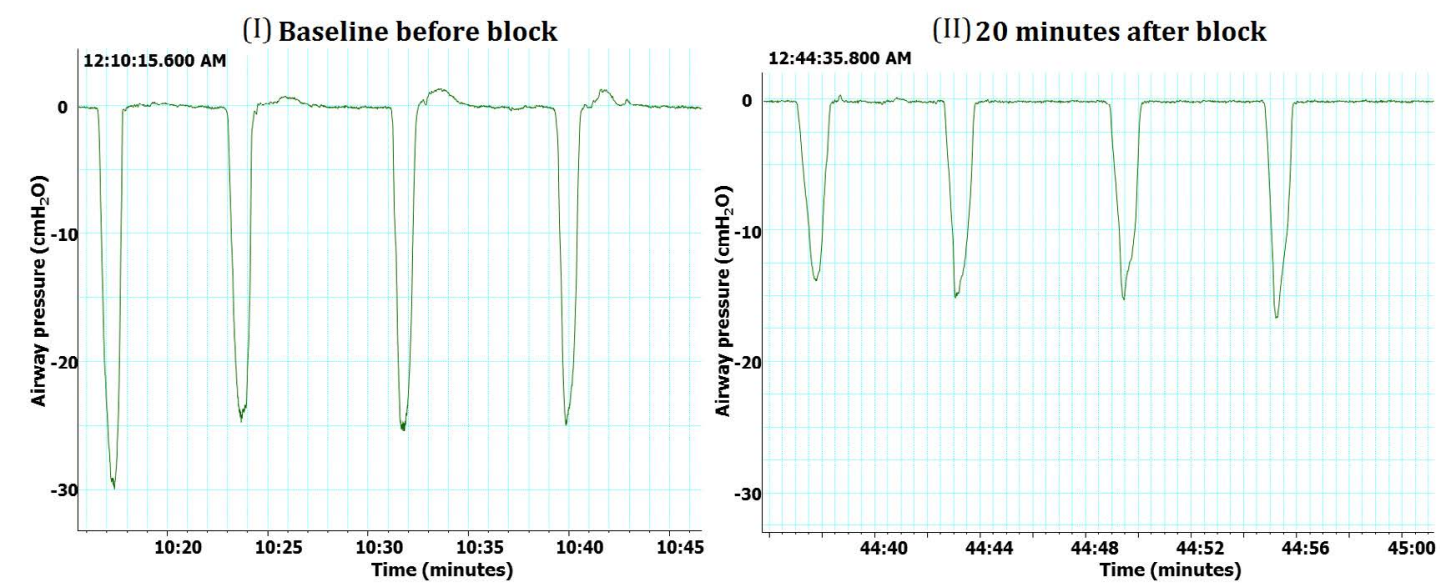

(A)

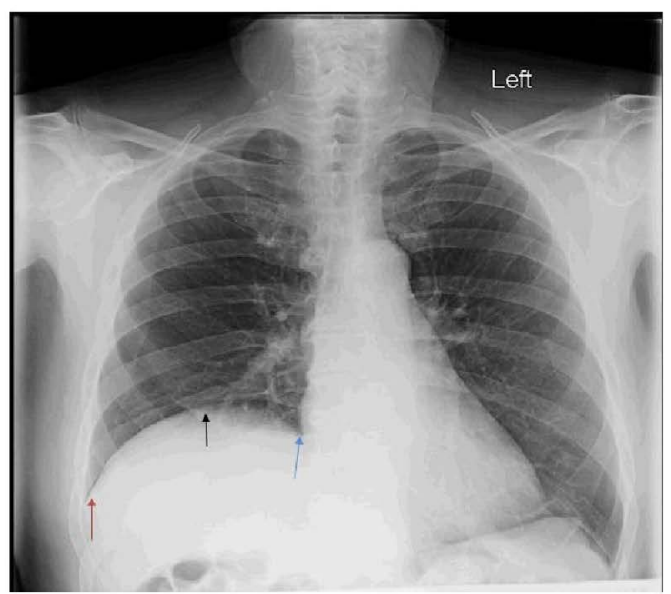

(B)

Figure 3A: Airway pressure waveforms before (I) and after (II) SCB using $30 \mathrm{cc}$ of ropivacaine $0.5 \%$. At pre-block (A, I), the patient was moving 3 balls up during incentive spirometry trials $(1200 \mathrm{cc} / \mathrm{min})$ and was able to generate an airway pressure of $-25 \mathrm{~cm}$ $\mathrm{H}_{2} \mathrm{O}$ on average. After 20 minutes post-block (A, II) patient was only able to move 1.5 balls on average $(<900 \mathrm{cc} / \mathrm{min})$ and to generate an airway pressure of $-15 \mathrm{~cm} \mathrm{H}_{2} \mathrm{O}$ on average.

Figure 3B: Anterior/posterior chest radiograph showing right hemi-diaphragmatic paresis (arrows) caused by local anesthetic influences on the phrenic nerve in a 46 yo male patient who received $30 \mathrm{cc}$ of $0.5 \%$ ropivacaine during a right supraclavicular block. 
Citation: Hesham ME, Dikstein AF, Rayaz H, et al. (2021) Interscalene Versus Supraclavicular Approach to Brachial Plexus Nerve Blockade and Local Anesthetic Volume Effect on the Incidence of Phrenic Nerve Dysfunction during Shoulder Surgery. J Clin Anesth Pain Manag 5(2):250-260

nerve involvement, determined as phrenic nerve blockade and resulting in hemidiaphragm paresis/dysfunction and then confirmed by both 1.) A reduced and compromised air inhalation ability on auscultation; as well as 2.) Performing a chest radiograph and examining the diaphragm for evidence of hemidiaphragm elevation (Figure 3B). Adverse LA effects on the phrenic nerve while performing a brachial plexus nerve block was defined as a $30 \%$ or greater reduction in the average number of plastic balls elevated on the IS trial with deep breathing during the 12 IS attempts of each trial conducted 20 minutes following plexus block completion and then compared to baseline values. The $30 \%$ or greater reduction on IS ball elevation value was an observational correlation between patients who clinically demonstrated adverse phrenic nerve involvement that was subsequently confirmed by lung auscultation and/or radiographic imaging of the lungs/diaphragm. Another post brachial plexus nerve block parameter identified was the presence or absence of mid-axillary chest pain on the side of the plexus nerve block during attempts at deep inspiration. Duration of the nerve block was determined by time in minutes and based on the patient's indication of retrurning sensory (tingling, pain, tempreture or touch) and then motor sensations of the blocked extremity that was determined and recorded when interviewing patients directly or by follow-up phone conversation(s).

A secondary outcome aspect from the investigation was to examine the process of validation of the IS device as a simple and non-invasive tool and mechanism for evaluating phrenic nerve involvement along with clinical influences that are possible when performing a brachial plexus nerve blockade. Additional secondary outcome data collected during the investigation included duration (in minutes) of the analgesic (sensory) components of the nerve/plexus block. Duration of analgesia was collected by comparing pre-block VAS pain scores to post-block VAS scores along with any evidence of LA systemic toxicity. These study parameters of post-block VAS scores were reported by patients during an elapsed24 hour/ next day phone conversation (for the outpatient surgical procedures) or by 24 hour/next day direct patient encounters (i.e. "rounding") with those patients admitted to the hospital following surgery and prior to hospital discharge.

\section{Statistical Analysis}

Numerical data (LA volume and duration) is presented as Mean (SD) and linear regression. Analysis of categorical data including 1) the incidence of phrenic involvement along with 2) the type of block being performed and 3) following stratification according to the volume of local anesthetic administered during each brachial plexus block being performed was evaluated and analyzed by the Chi-square test. Comparing demographic data, VAS pain scores and average number of IS balls elevated by patients was analyzed using Mann Whitney Rank sum test. $P$-value $<0.05$ was accepted as statistically significant.

\section{Results}

All 155 patients receiving a brachial plexus peripheral nerve block (PNB) for shoulder surgeries (including reverse total shoulder, total shoulder arthroplasty, arthroscopy, rotator cuff repair, arthrodesis, reconstruction, internal fixation and labral repair) were initially divided into two groups depending upon the PNB approach utilized; either a SCB $(n=88)$ or an ISB $(n=67)$ approach to the brachial plexus. Each of the 2 groups were further divided according to the volume of LA being administered during PNB performance; sub divided into either a high volume (HV) subgroup or low volume (LV) subgroup of LA being injected and then defined as greater than a $20 \mathrm{ml}$ volume (HV) or a $20 \mathrm{ml}$ volume or less (LV) respectively. Among the 88 patients who received a SCB approach to the brachial plexus, 39 received $\mathrm{HV}$ and 49received LV of LA. Of the 67 study subjects who experienced an ISB approach to the brachial plexus; 38 had injected a HV of local anesthesia while 29received the LV of local.

There were no statistical differences in the demographics or PNB outcome characteristics among the groups and subgroups (Table 1). At baseline, prior to PNB placement and LA injection, the average Visual Analogue Scale (VAS) pain scores without movement were 2.5 (2.9) in the SCB group and 1.5 (2.4) for the ISB group ( $p=0.212$ ) but with movement, VAS values of 6.6 (3.1) for the SCB and 5.5 (3.5) in the ISB group $(p=0.25)$. None of the patients in either group or within the 4 subgroups experienced any residual pain 20 minutes following PNB placement. There was no subjective or objective evidence nor significant difference between the number of IS balls elevated prior to PNB placement in either group or between subgroups. This observation therefore, is reflective of comparable baseline respiratory mechanics and function between both the SCB and ISB groups [average (SD) was 2.7(0.5) for the SCB and $2.8(0.3)$ in the ISB group ( $p=$ 0.667)].

All patients from both groups experienced successful nerve/plexus blocks as evidenced by neurological examination within 20minutes following PNB placement (using an alcohol swab and pinprick to compare sensations within dermatomal distribution of blocked and contralateral extremities). Incidence of phrenic nerve involvement/dysfunction in those patients receiving an ISB was $68.7 \%$ (46 out of 67 ) versus $26.1 \%$ (23 out of 88 ) among patients received a SCB ( $p<$ 0.001 ) (Figure $4 A$ ). Within the ISB group when subdivided by volume of LA administered, the incidence of phrenic nerve dysfunction was $79 \%$ (30 out of 38 ) and $55.2 \%$ (16 out of 29) in the HV and LV subgroups respectively ( $p=0.038)$. In the SCB group, the incidence of phrenic nerve dysfunction was $35.9 \%$ (14 out of 39 ) and $16.3 \%$ (8 out of 49 ) in the HV and LV subgroups respectively $(p=0.035)$ (Figure 4B).

Duration of PNB effectiveness/analgesia reported by the study patients revealed no significant difference between SCB and ISB groups and no differences in duration of effect between either the HV or LV subgroups. The average LA volumes administered was $23.7 \mathrm{ml}$ (7.4) and $24.7 \mathrm{ml}$ (8) for SCB and ISB groups respectively and average duration ( $\mathrm{hr}$ ) achieved was 14.7(4.5) and 14.1 (4.5) for the SCB and ISB groups respectively as shown in (Figure 4 C, Table 2). None of the patients in the investigation showed any clinical signs or symptoms of systemic LA toxicity. 

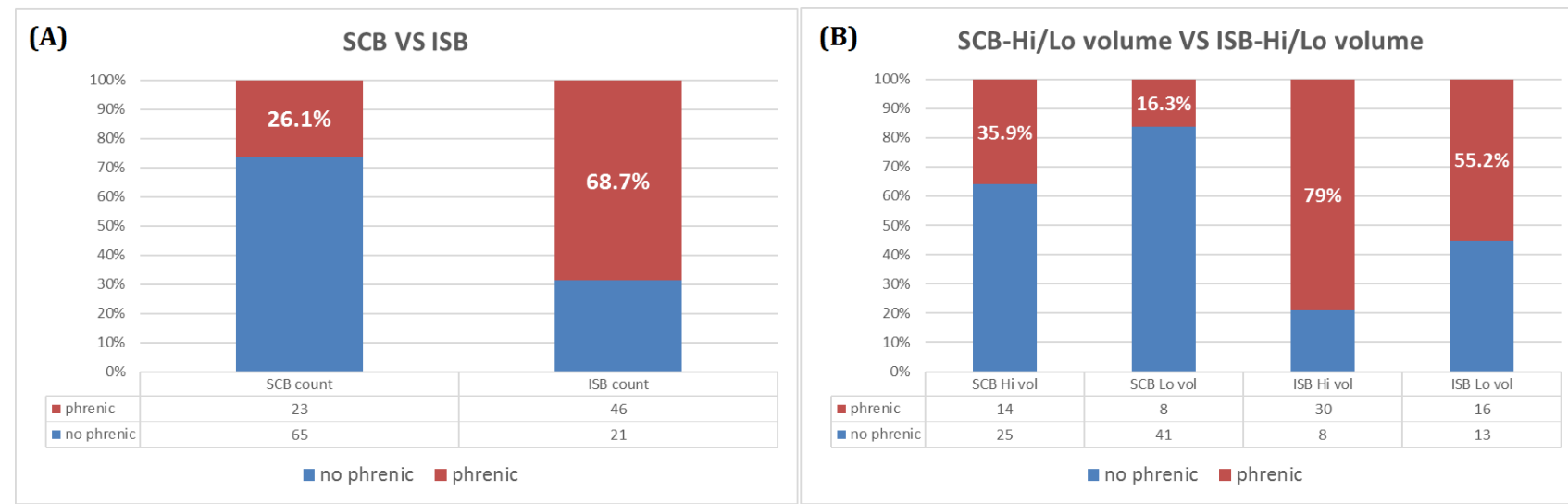

(C)

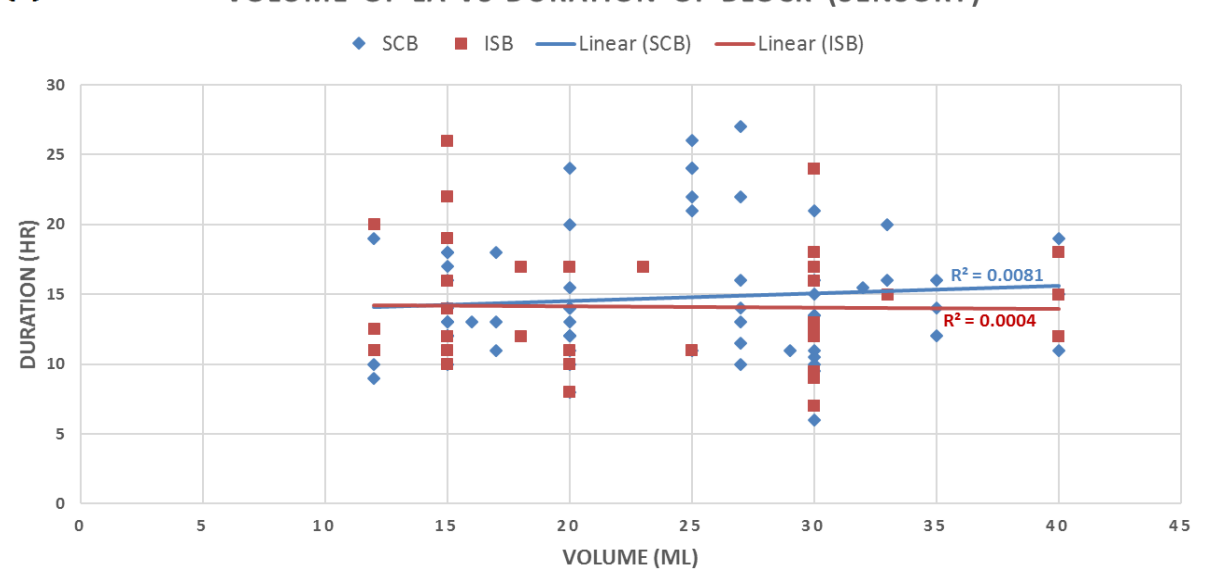

Figure 4A: Incidence of phrenic nerve involvement with ISB vs. SCB.

Figure 4B: Incidence of phrenic nerve involvement with different volumes of local anesthetic in ISB and SCB.

Figure 4C: Effects of LA volumes on the duration of sensory blockade.

Table 1: Comparable demographic data between both groups.

\begin{tabular}{|c|c|c|c|c|c|}
\hline & & SCB $(\boldsymbol{n}=\mathbf{8 8})$ & ISB $(\boldsymbol{n}=\mathbf{6 7})$ & TOTAL $(\boldsymbol{n}=\mathbf{1 5 5})$ & p-value \\
\hline Age (years) & Mean (SD) & $52(15.5)$ & $51.8(18.2)$ & $52(16.5)$ & 0.997 \\
\hline & Range & $20-83$ & $18-82$ & $16-83$ & 0.952 \\
\hline BMI (Kg/m $\mathbf{2})$ & Mean (SD) & $29.9(8)$ & $29.7(8)$ & $29.8(7.9)$ & $15.1-67.6$ \\
\hline & Range & $19.3-67.6$ & $15.1-58.7$ & $53 \%$ & \multirow{2}{*}{0.902} \\
\hline
\end{tabular}

Table 2: Effects of LA volumes on the duration of sensory blockade.

\begin{tabular}{|c|c|c|c|c|}
\hline & & Volume (ml) & Duration (hr) & $\mathbf{R}^{2}$ \\
\hline & Mean (SD) & $24.7(8)$ & $14.1(4.5)$ & 0.0004 \\
\hline & Range & $12-40$ & $7-26$ & 0.0081 \\
\hline
\end{tabular}

\section{Study Limitations}

A limitation of thestudy was a lack of consistant blinding/ blindednessamong the 5 physicians performing the PNBand amongthe research team members collecting the datain regards to a) the PNB approach taken (i.e either ISB or SCB); b) volume of local anesthetic administered during PNB performance (i.e. HV or LV of injectate); c) number of block needle passes; d) number of locations within the choosen approach (SCB or ISB) in which local was injected; e) proximity to the subclavian artery during either ISB or SCB approach choosen; and f) an inability to blind the data collectors from being able to observe/witness the needle insertion site of the choosen injection approach (ISB or SCB). Another limitation was related to determining the precise or exact (to the minute) duration of effect for each brachial plexus nerve block 
Citation: Hesham ME, Dikstein AF, Rayaz H, et al. (2021) Interscalene Versus Supraclavicular Approach to Brachial Plexus Nerve Blockade and Local Anesthetic Volume Effect on the Incidence of Phrenic Nerve Dysfunction during Shoulder Surgery. J Clin Anesth Pain Manag 5(2):250-260

performedas this parameter of cessation of effects from neural blockade is most often gradual in occurrence and was being reported by a patients expression or return of "feelings" (i.e pain and touch) andregainingof "normal"sensation(s) and/ or function of the blocked extremity (waning of the PNB effectiveness). This study only investigatedshoulder surgery candidates receiving a single-shot brachial plexus nerve block and not for any other upper extremity surgery nor consideration(s) associated with an indwelling catheter. The use of an IS device as a diagnositic tool examining for any evidence of diaphragmatic compromise/hemiparesis due to LA influence on the phrenic nerve used in this investigationwas not validated and not compared to M-mode ultrasound [21]. Additional limitations of the investigation included data regarding intraoperative general anesthesia requirements, exact timing of postoperative opiate use/requirements/ administration, and postoperative congnitive dysfunction secondary to opioid use and/or misuse were not recorded.

\section{Discussion}

A most significant finding from this study revealed that injecting $\leq 20 \mathrm{ml}$ of $0.5 \%$ ropivacaine under direct US-guidance during the SCB approach of a brachial plexus nerve blockade for shoulder surgery could provide optimal and effective perioperative analgesia with a statistically significant lower incidence of phrenic nerve compromise and dysfunction. Comparing the SCB approach of plexus nerve blockade and injecting $\leq 20 \mathrm{ml}$ of LA to the ISB approach and/or using higher LA volumes (greater than $20 \mathrm{ml}$ ) provides optimal analgesic success and efficacy without compromising either quality and/or duration of brachial plexus nerve blockade. This study also showed that the use of an IS device was easy to use and reliable, inexpensive, and an objective mechanism to examine and assess the effect (s) of inspiratory capacity following completion of a proximal (ISB or SCB) brachial plexus nerve block inpatients scheduled for shoulder surgery. However, additional studies are warranted and would be necessary to continue to validate the sensitivity and specificity of IS versus the current "gold standard" provided by M-mode ultrasonography when assessing phrenic nerve compromise and resultant diaphragmatic function following proximal (ISB or SCB) brachial plexus blockade performed during upper extremity surgery.

The literature has identified and suggested that certain insightful measures and practice techniques can be implemented to curtail the incidence of potential deleterious consequences of phrenic nerve compromise and dysfunction related to LA effects resulting from the performance of proximal (ISB and SCB) brachial plexus blockade during shoulder surgery $[10,12,15-20]$. The results from this investigation were also able to confirm several of the performance measures indicated within the literature that can reduce phrenic nerve compromise and insult during proximal brachial plexus nerve blockade such as: 1) strategic needle tip placement within the neurovascular bundle; 2) administering lower volumes $(\leq 20 \mathrm{ml})$ of $L A ; 3)$ altering the approach(ISB versus SCB) used when performing a brachial plexus block; 4) applying pressure cephalad to the chosen brachial plexus injection site during LA administration; and 5) relying on ultrasound (US)-guided RA to visualize the injected LA spread. The more distal and lateral anatomical location of the SCB approach to brachial plexus (i.e. divisions) blockade from the phrenic nerve (typically on the anterior surface of the scalenus anterior muscle) when relatively compared to the ISB approach can result in a lowered incidence of negative influences toward phrenic nerve compromise/blockade when performing a SCB technique versus an ISB approach/ technique $[8,9]$.

Recent reports within the literature have also recommendedadministeringlower volumes and/or concentrations of LA and to target a more distal aspect/ component of the plexus with intent toward achieving less phrenic nerve dysfunction following brachial plexus nerve blockade [17]. According to a study by Auyong, et al; the authors showed reduced phrenic nerve dysfunction, comparable postoperative pain control and opioids analgesic requirements, pain management effectiveness that compared favorably to both SCB and ISB approaches, along with preserved post-block vital capacity when performing a suprascapular (innervates $\sim 75 \%$ of the shoulder joint) nerve block for shoulder surgery compared to both SCB and ISB techniques [18]. In addition, administering lower volumes of LA under direct US-guidance and real-time visualization of its spread around the target structures proved beneficial in minimizing adverse neurologic side effects [22].

Several of the regional block protocols, pearls, and techniques mentioned above that were used in an attempt to reduce the incidence of adverse phrenic nerve influence will enhance the perioperative pain medicine experience provided to shoulder surgery patients. However, implementing a brachial plexus block approach that can eliminate deleterious phrenic nerve impact can especially benefit those patient with compromised respiratory function such as chronic obstructive sleep apnea (COPD), chronic smokers, obese patitents and patients with history of obstructive sleep apnea or obesity hypoventilation syndrome. Negative short-term consequences or neusence symptoms from phrenic nerve dysfunction are usually well tolerated by most patients that have been consented and educated about these possible side effects. However,for patients with compromised respiratory status or limited inspiratory reserve; any added pulmonary function insult or negative influence(s) on respiratory mechanics can lead to more serious symptomatic respiratory distress, decompensation and respiratory failure [14]. Another more serious and potential for negative longerterm complications that can be experienced by all patients is the occurrence of chronic phrenic nerve dysfunction or neurotoxic compromise.

Chronic phrenic nerve dysfunction following abrachial plexus nerve blockade is theorized to be due to direct LA toxicity, perineural inflammation, and fibrosis that can result in nerve entrapment and chronic dysfunction that may require surgical treatment in order to improve any negative diaphragm functions [4-6]. It has also been suggested that patients with symptomatic cervical spine pathology may be at an increased risk for developing chronic phrenic nerve dysfunction after brachial plexus blockade [5], but further 
Citation: Hesham ME, Dikstein AF, Rayaz H, et al. (2021) Interscalene Versus Supraclavicular Approach to Brachial Plexus Nerve Blockade and Local Anesthetic Volume Effect on the Incidence of Phrenic Nerve Dysfunction during Shoulder Surgery. J Clin Anesth Pain Manag 5(2):250-260

investigation is required to study this incidence and to confirm any additional risk factors for the types of phrenic nerve injury following brachial plexus nerve blockade.

\section{Conclusions}

This investigation revealed that the unconventional or atypical SCB approach during brachial plexus nerve blockadetoachieve postoperativeanalgesiafollowingshoulder surgery with LA volumes of $20 \mathrm{ml}$ or less can provide optimal clinical efficacy and perioperative pain management compared to the ISB approach. It was also shown that the SCB approach was just as effective as the more conventional ISBto the brachial plexus (targeting C5, C6 and C7 area), but with a statistically significant lower incidence of phrenic nerve dysfunction when the volumes of injected LA were $20 \mathrm{ml}$ or less. The negative side effect profile on diaphragm function during brachial plexus nerve blockade (regardless of approach taken; ISB or SCB) using higher volumes (i.e. $>20 \mathrm{ml}$ ) of LA carries a greater risk of adverse consequences without any added benefit or prolongation of nerve plexus block analgesic duration. This is especially important to consider, and implement a change in practice; in those patients with compromising pulmonary function or reserve where adverse phrenic nerve influences could result in serious compromise to respiratory mechanics.

In addition, this investigation revealed the value and ease of implementing an additional tool, the easy to use IS device, toward evaluating phrenic nerve influences and its potential compromise of diaphraghm functioning. The use of an IS as a simple device to monitor diaphragm influences due to phrenic nerve LA insult when performing abrachial plexus nerve block and subsequent effects on respiratory function and mechanics has been shown to be a reliable and inexpensive option.

However, additional investigation is needed to further confirm and authenticate that using alow volume ( $\leq 20 \mathrm{ml}$ ) of LA when practicing a SCB approach to brachial plexus blockade, as described in this study, could validate and endorse this more optimal SCB technique during performance of abrachial plexus nerve block for analgesia during shoulder surgery. These additional studies will substantiate the SCB approach before advocating for a change in the "standard of care". Performing additional investigation will also contribute to the fund of knowledge and futher authenticate the values of injecting smaller volumes of LA during a SCB approach to the brachial plexus resulting in a reduction and/or elimination toward adverse phrenic nerve influences. In addition, further studies are also necessary to assess the sensitivity and specificity of the IS device as a screening tool or technique to validate its ability when objectively evaluating for any evidence of phrenic nerve compromise or dysfunction following a brachial plexus nerve block.

\section{Conflicts of Interests/Financial Disclosures}

\section{None.}

\section{Meetings}

Presented in ASRA 15th Annual Pain Medicine Meeting, November 2016 (https://www.asra.com/content/docu- ments/2861_interscalene-versus-supraclavi_1609300827. pdf) and awarded "Best of Meeting" with oral presentation at the best of meeting awards luncheon (https://www.asra. com/page/1330/best-of-meeting-awards).

\section{Authors' contribution}

Hesham M Ezz MB ChB: This author is the first, assisted in collecting data, performed data analysis, and presenting the topic in the ASRA 2016 Pain Medicine meeting.

Anna F Dikstein MS-3: This author helped in collecting data, data analysis and reviewing the manuscript.

Mohamed F El Meliegy MD: This author helped in collecting data, data analysis.

Hassan Rayaz MD: This author helped in collecting data and reviewing the manuscript.

Elissa Mastrangelo MS, BC: This author helped in collecting data and reviewing the manuscript.

Michael J Medvecky MD: This author helped in reviewing the manuscript.

Thomas M Halaszynski DMD, MD, MBA: This author helped in designing the protocol, collecting data, results interpretation, writing and reviewing the manuscript. He is also serving as the corresponding author.

Aymen A Alian MD: This author helped by designing the protocol, collecting data, results interpretation and reviewing the manuscript.

\section{References}

1. Klein SM, Evans H, Nielsen KC, et al. (2005) Peripheral nerve block techniques for ambulatory surgery. Anesth Analg 101: 1663-1676.

2. Neal JM, Gerancher JC, Hebl JR, et al. (2009) Upper extremity regional anesthesia: Essentials of our current understanding, 2008. Reg Anesth Pain Med 34: 134-170.

3. Franco CD, Vieira ZE (2000) 1,001 subclavian perivascular brachial plexus blocks: success with a nerve stimulator. Reg Anesth Pain Med 25: 41-46.

4. Hogan QH (2013) Phrenic nerve function after interscalene block revisited: Now, the long view. Anesthesiology 119: 250252.

5. Pakala SR, Beckman JD, Lyman S, et al. (2013) Cervical spine disease is a risk factor for persistent phrenic nerve paresis following interscalene nerve block. Reg Anesth Pain Med 38: 239-242.

6. Kaufman MR, Elkwood Al, Rose MI, et al. (2013) Surgical treatment of permanent diaphragm paralysis after interscalene nerve block for shoulder surgery. Anesthesiology 119: 484-487.

7. Dhuner KG, Moberg E, Onne L (1955) Paresis of the phrenic nerve during brachial plexus block analgesia and its importance. Acta Chir Scand 109: 53-57.

8. Knoblanche GE (1979) The incidence and aetiology of phrenic nerve blockade associated with supraclavicular brachial plexus block. Anaesth Intensive Care 7: 346-349. 
Citation: Hesham ME, Dikstein AF, Rayaz H, et al. (2021) Interscalene Versus Supraclavicular Approach to Brachial Plexus Nerve Blockade and Local Anesthetic Volume Effect on the Incidence of Phrenic Nerve Dysfunction during Shoulder Surgery. J Clin Anesth Pain Manag 5(2):250-260

9. Urmey WF, Talts KH, Sharrock NE (1991) One hundred percent incidence of hemidiaphragmatic paresis associated with interscalene brachial plexus anesthesia as diagnosed by ultrasonography. Anesth Analg 72: 498-503.

10. Renes SH, Spoormans HH, Gielen MJ, et al. (2009) Hemidiaphragmatic paresis can be avoided in ultrasoundguided supraclavicular brachial plexus block. Reg Anesth Pain Med 34: 595-599.

11. Renes SH, Rettig HC, Gielen MJ, et al. (2009) Ultrasoundguided low-dose interscalene brachial plexus block reduces the incidence of hemidiaphragmatic paresis. Reg Anesth Pain Med 34: 498-502.

12. Urmey WF, McDonald M (1992) Hemidiaphragmatic paresis during interscalene brachial plexus block: Effects on pulmonary function and chest wall mechanics. Anesth Analg 74: 352-357.

13. Ediale KR, Myung CR, Neuman GG (2004) Prolonged hemidiaphragmatic paralysis following interscalene brachial plexus block. J Clin Anesth 16: 573-575.

14. Hood J, Knoblanche G (1979) Respiratory failure following brachial plexus block. Anaesth Intensive Care 7: 285-286.

15. Casati A, Fanelli G, Cedrati V, et al. (1999) Pulmonary function changes after interscalene brachial plexus anesthesia with $0.5 \%$ and $0.75 \%$ ropivacaine: A double-blinded comparison with $2 \%$ mepivacaine. Anesth Analg 88: 587-592.
16. Vandepitte C, Kuroda M, Witvrouw R, et al. (2017) Addition of Liposome Bupivacaine to Bupivacaine $\mathrm{HCl}$ Versus Bupivacaine $\mathrm{HCl}$ Alone for Interscalene Brachial Plexus Block in Patients Having Major Shoulder Surgery. Reg Anesth Pain Med 42: 334341.

17. Urmey WF, Gloeggler PJ (1993) Pulmonary function changes during interscalene brachial plexus block: effects of decreasing local anesthetic injection volume. Reg Anesth 18: 244-249.

18. Chin KJ, Chan V (2016) Tactics for reducing risk for interscalece block-related phrenic nerve palsy. Anesthesiology news 10.

19. Auyong DB, Yuan SC, Choi DS, et al. (2017) A Doubleblind randomized comparison of continuous interscalene, supraclavicular, and suprascapular blocks for total shoulder arthroplasty. Reg Anesth Pain Med 42: 302-309.

20. Urmey WF, Grossi P, Sharrock NE, et al. (1996) Digital pressure during interscalene block is clinically ineffective in preventing anesthetic spread to the cervical plexus. Anesth Analg 83: 366370.

21. Testa A, Soldati G, Giannuzzi R, et al. (2011) Ultrasound M-mode assessment of diaphragmatic kinetics by anterior transverse scanning in healthy subjects. Ultrasound Med Biol 37: 44-52.

22. Smith HM, Duncan CM, Hebl JR (2009) Clinical utility of low-volume ultrasound-guided interscalene blockade: Contraindications reconsidered. J Ultrasound Med 28: 1251-1258.

DOI: $10.36959 / 377 / 363$

Copyright: (C) 2021 Hesham ME. This is an open-access article distributed under the terms of the Creative Commons Attribution License, which permits unrestricted use, distribution, and reproduction in any medium, provided the original author and source are credited. 